Check for updates

The BMJ

Cite this as: $B M J 2020 ; 370: m 3585$ http://dx.doi.org/10.1136/bmj.m3585 Published: 16 September 2020

\section{Operation Moonshot: Testing plan relies on technology that does not exist}

\section{Elisabeth Mahase}

Delivering mass testing on the scale and level of ambition set by the UK prime minister, Boris Johnson, will probably require "testing technology that currently does not exist," say leaked documents revealed by The BMJ. ${ }^{1}$

The Operation Moonshot plans, which could see the government spend over £1oobn to ensure 10 million covid-19 tests a day, show it's likely that new testing technology would need to be developed, validated, procured, and made operational within months to meet the early 2021 deadline.

Jon Deeks, professor of biostatistics at the University of Birmingham and leader of the Cochrane Collaboration's covid-19 test evaluation activities, has described the plan as a "nice dream."

He told The BMJ, "This is not the way we should be tackling something when people are dying right now: thinking about things we have not got. We should be thinking about the things we have got and we know work. Backing a horse that hasn't yet been born is a really bad strategy."

The Moonshot documents list several covid-19 tests being considered for rollout across the UK, despite some having lower sensitivity than polymerase chain reaction (PCR) tests. These would be used "for screening/enabling purposes, with PCR used to confirm positive results or in situations where accuracy is needed for highest risk individuals," say the documents.

\section{What do we know about the tests?}

The tests listed in the documents are reverse transcriptase (RT) PCR, Endpoint PCR, LamPORE, Direct LAMP, lateral flow antigen tests, and whole genome sequencing. However, there is not much publicly available evidence for the use of these tests on SARS-CoV-2. What is accessible is mainly from non-peer reviewed preprints of research carried out by the manufacturers.

For example, in the case of RT-PCR a preprint paper from the company DnaNudge claimed that its point-of-care test, which involves nasopharyngeal swabs, had an average sensitivity of $94.4 \%$ (95\% confidence interval $86 \%$ to $98 \%$ ) and an overall specificity of $100 \% .^{2}$ It concluded that the test was "specific and rapid" and could detect SARS-CoV-2 "without laboratory handling or sample pre-processing."

A study assessing LamPORE (also available as a preprint), carried out by its manufacturer, Oxford Nanopore Technologies, claimed that the test was "rapid, sensitive, and highly scalable."3 It reported that results could be obtained from 12 samples in about an hour, when starting with extracted RNA, and from 96 samples in less than two hours. It also said that the test correctly identified 79 of 80 positive samples.

However, Deeks pointed out some inaccuracies in the documents concerning the proposed tests. He said, "I have spoken to the person doing the DnaNudge evaluation, and he said they have not done it on saliva-they have only done it on swab [samples]. So why has that list got saliva on it?"

Also, Deeks said that, despite LamPORE being listed as taking 90 minutes, it actually needs 6.5 hours to run. "The 90 minutes is the middle bit. That does not include plating up the samples, doing the DNA extraction, and things like that. So from sample to result it's about 6.5 hours."

He added, "No publicly available data for a lot of the tests [are available] ... It's not good science. If these were drugs, the government would have had to register these studies on the Clinical Trials Register, with the protocol, and to publish the results."

\section{Potential harms}

Deeks's main concern was that the "whole of this programme has been built without thinking about the harms it could do.”

He said, "The mathematical laws as to what happens when you start screening mass populations are not in the right direction in terms of the harms that will be done. The key thing is specificity, and the documents do not mention specificity or false positives, which is how you get harm. If you start using tests in 60 million people, even if they are $99 \%$ specific you will end up giving false positive results to hundreds of thousands of people."

Deeks warned that using less reliable tests to enable people to access events such as football matches would be "dangerous," as it could give people false confidence and see them ease protective measures such as social distancing. 
However, he was positive about expanding testing, provided that it was done correctly. "Some of these tests have potential to improve how well we can do test and trace. If they can improve the capacity and speed up the time, that would be very useful, because we need that right now. We just need to be very clear as to where they are going to go and what their purpose is," he said.

\section{What is happening in Greater Manchester?}

The Moonshot documents emphasise that trialling new models for delivering testing in local areas will be a "critical part" of the strategy. This includes a large trial in Salford, Greater Manchester, where the prevalence of covid-19 is currently higher than in most of the UK.

The Salford City Council website says that the trial involves weekly testing of the saliva of people who do not have covid-19 to "identify any positive cases early and allow those who know they are coronavirus free to go about their normal lives." 4

The test being used is the LAMP test, and though the council has said it will be rolled out in phases across the area, it gives no dates for when this will happen.

A statement on the website says, 'It is proposed that there will be an initial two-week period to test the 'proof of concept.' This would enable us to test end-to-end the reliability of the different elements of what is a complex practical process. The 'proof of concept' testing will take place with smaller groups of our community and enable us to ensure each of these elements is working well before then rolling out a Salford-wide programme, that is yet to be determined."

1 lacobucci G, Coombes R. Covid-19: Government plans to spend f100bn on expanding testing to 10 million a day. BM/2020;370:m3520. doi: 10.1136/bmj.m3520 pmid: 32907851

2 Gibani MM, Toumazou C, Sohbati M, et al. CovidNudge: diagnostic accuracy of a novel lab-free point-of-care diagnostic for SARS-CoV-2. MedRxiv. 15 Aug 2020. https://www.medrxiv.org/content/10.1101/2020.08.13.20174193v1.full.pdf.

3 James P, Stoddart D, Harrington ED, et al. LamPORE: rapid, accurate and highly scalable molecular screening for SARS-CoV-2 infection, based on nanopore sequencing. MedRxiv. 7 Aug 2020. https://www.medrxiv.org/content/10.1101/2020.08.07.20161737v1.

4 Salford City Council. Community testing. https://www.salford.gov.uk/people-communities-and-local-information/coronavirus/help-for-residents/community-testing.

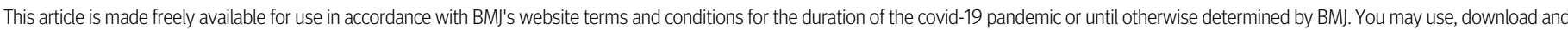
print the article for any lawful, non-commercial purpose (including text and data mining) provided that all copyright notices and trade marks are retained. 